\title{
Airborne Multistatic Polarimetric Radar Modeling
}

\author{
Tegan Webster \\ Radar Division \\ Naval Research Laboratory \\ Washington, DC, USA
}

\begin{abstract}
Previous work developed a data model and corresponding imaging operation for multistatic polarimetric radar with stationary transmitters and receivers and moving targets. This paper extends the model to an airborne multistatic polarimetric radar scenario and considers multiple moving transmitting and receiving platforms in addition to moving targets. The formulation results in an expression for the received data that may be coherently combined across receivers, represented in a global coordinate system.
\end{abstract}

\section{INTRODUCTION}

Multistatic radar with distributed transmitters and/or receivers is capable of examining a target from multiple spatial angles, possibly utilizing different waveforms and different frequencies. Similarly, polarimetric radar can examine a target with multiple transmit and/or receive polarizations, yielding the potential to reconstruct the full scattering matrix of the target. The scattering response of a target varies with aspect angle, frequency, and polarization and therefore multistatic polarimetric radar offers the potential to gain more information about a target relative to single-polarization monostatic radar.

Related work within the area of multistatic radar data modeling, detection, and imaging includes adaptive processing for distributed aperture radar [1], [2], signal processing for multistatic radar [3], multistatic imaging of a stationary scene in a multipath environment [4], scalar mathematical modeling and imaging of multistatic radar data [5]-[7], and polarimetric modeling and imaging of multistatic radar data [8]. Additional work in polarimetric radar data modeling with moving platforms has considered stationary targets with monostatic active sensors [9] and multistatic passive sensors [10]. This work addresses multistatic polarimetric radar data modeling for an airborne moving target indicator (MTI) scenario.

Prior work developed a vector radar data model and corresponding imaging operations for multistatic polarimetric radar with stationary transmitters and receivers and distributions of non-interacting moving targets [8]. This work extends [8] by formulating a full vector data model for multistatic polarimetric radar with moving platforms and moving targets. Specifically this work outlines the formulation of the data model and results in an expression for data obtained at each receiver that can be coherently processed across receivers, represented in a global coordinate system.

\section{Multistatic Scenario}

The multistatic scene consists of $M$ transmitters indexed $m=1, \ldots, M$ at position $\gamma_{\mathrm{T}, m}(s), N$ and receivers indexed $n=1, \ldots, N$ at position $\gamma_{\mathrm{R}, n}(s)$, where $s$ is a coarse time variable. The coarse time variable is used to define the positions and velocities of transmitters and receivers relative to the start of the interval of time during which data from the bistatic pairs is coherently processed. The model assumes that for the application of multistatic MTI and consistent radar parameters, the positions and velocities of transmitters and receivers can be defined once for the time interval of coherently processed data. The moving target of interest is located at position $\boldsymbol{x}_{a}$ and has velocity $\boldsymbol{v}_{a}$.

All antennas in the scene are modeled as long thin dipoles of length $2 L$ and diameter $2 \alpha$. The data model utilizes a target-centered backscatter alignment (BSA) convention global coordinate system relating the $m^{\text {th }}$ transmitter at time $s, n^{\text {th }}$ receiver at time $s$, and target. Each bistatic pair associated with a target requires a unique coordinate system.

\section{DATA MODEL}

As in previous work, the electric field radiated from the $m^{\text {th }}$ transmitter, denoted $\boldsymbol{E}_{m}^{\mathrm{t}}$, is obtained by taking the farfield portion of the frequency-domain electric field that arises from the potential formulation of Maxwell's equations and the Green's function solution to the wave equation. This far-field portion is

$$
\begin{aligned}
\boldsymbol{E}_{m}^{\mathrm{t}}\left(\boldsymbol{r}_{\mathrm{T}, m}, \omega, s\right)= & -\mathrm{i} \omega \mu_{0} \frac{\mathrm{e}^{\mathrm{i} k\left|\boldsymbol{r}_{\mathrm{T}, m}\right|}}{4 \pi\left|\boldsymbol{r}_{\mathrm{T}, m}\right|} \\
& \cdot\left(\hat{\boldsymbol{r}}_{\mathrm{T}, m} \times\left(\hat{\boldsymbol{r}}_{\mathrm{T}, m} \times \boldsymbol{F}\left(k \hat{\boldsymbol{r}}_{\mathrm{T}, m}, \omega\right)\right)\right)
\end{aligned}
$$

where

$$
\boldsymbol{F}_{\mathrm{T}, m}\left(k \hat{\boldsymbol{r}}_{\mathrm{T}, m}, \omega\right)=\int \mathrm{e}^{-\mathrm{i} k \hat{\boldsymbol{r}}_{\mathrm{T}, m} \cdot \boldsymbol{r}_{\mathrm{T}, m}^{\prime}} \boldsymbol{J}\left(\boldsymbol{r}^{\prime}, \omega\right) \mathrm{d} \boldsymbol{r}_{\mathrm{T}, m}^{\prime}
$$

denotes the radiation vector, the spatial Fourier transform of the frequency-domain current density $\boldsymbol{J}$, and

$$
\boldsymbol{r}_{\mathrm{T}, m}=\boldsymbol{x}-\gamma_{\mathrm{T}, m}(s)
$$

where $\boldsymbol{x}$ is a location in space. As in [8], the frequencydomain transmit waveform is included in the expression for the radiation vector $\boldsymbol{F}_{\mathrm{T}, m}$ within the current density term. It is possible that the transmit waveforms of the $M$ transmitters will not be synchronized in time or share the same pulse repetition frequency, number of pulses, or pulse width. In this model it is assumed that the interval of time for which the data from all bistatic pairs will be coherently processed begins at time $s$. Additionally, $S_{m}(\omega, s)$ defines the Fourier transform of a 
time-domain signal that is transmitted from the $m^{\text {th }}$ transmitter, begins at time $s$, and extends in time for the duration of the common coherent processing interval among the $M$ transmitters. Coherent processing requires accurate position and frequency information for all sensors in the system. If these conditions cannot be met, then the data obtained at each of the distributed receivers may be noncoherently processed as discussed in [8].

The scattered electric field at position $x$ from a moving target at location $\boldsymbol{x}_{a}$ is given by

$$
\begin{aligned}
\boldsymbol{E}_{m}^{\mathrm{r}}(\boldsymbol{x}, \omega, s)= & \frac{\mathrm{e}^{\mathrm{i} k\left|\boldsymbol{x}_{a}-\boldsymbol{x}\right|}}{4 \pi\left|\boldsymbol{x}_{a}-\boldsymbol{x}\right|}[\boldsymbol{S}]_{\boldsymbol{x}_{a}} \boldsymbol{E}_{m}^{\mathrm{t}}\left(\boldsymbol{r}_{\mathrm{T}, m}^{a}, \omega, s\right) \\
= & \mathrm{i} \omega \mu_{0} \frac{\mathrm{e}^{\mathrm{i} k\left(\left|\boldsymbol{r}_{\mathrm{T}, m}^{a}\right|+\left|\boldsymbol{x}_{a}-\boldsymbol{x}\right|\right)}}{(4 \pi)^{2}\left|\boldsymbol{r}_{\mathrm{T}, m}^{a}\right|\left|\boldsymbol{x}_{a}-\boldsymbol{x}\right|} F_{\mathrm{T}, m}\left(\psi_{\mathrm{T}, m}\right) \\
& \cdot\left([\boldsymbol{S}]_{\boldsymbol{x}_{a}} \hat{\boldsymbol{p}}_{\mathrm{T}, m}\right)
\end{aligned}
$$

where

$$
\boldsymbol{r}_{\mathrm{T}, m}^{a}=\boldsymbol{x}_{a}-\gamma_{\mathrm{T}, m}(s)
$$

is the vector from the $m^{\text {th }}$ transmitter at time $s$ to the $a^{\text {th }}$ target, $[\boldsymbol{S}]_{\boldsymbol{x}_{a}}$ is the BSA convention scattering matrix of the $a^{\text {th }}$ target, $F_{\mathrm{T}, m}$ is the scalar radiation pattern of the $m^{\text {th }}$ transmitting antenna, $\psi_{\mathrm{T}, m}$ is the angle between the vector defining the antenna orientation and the vector from the antenna to the target, and $\hat{\boldsymbol{p}}_{\mathrm{T}, m}$ is the unit vector containing the horizontal and vertical components of the antenna polarization in the basis of the antenna.

The open circuit received voltage at the $n^{\text {th }}$ receiver due to the $a^{\text {th }}$ target is

$V_{m n}^{\mathrm{r}}\left(\omega, s, \boldsymbol{x}_{a}, \boldsymbol{v}_{a}\right)=\boldsymbol{E}_{m}^{\mathrm{r}}\left(\gamma_{\mathrm{R}, n}(s), \omega, s\right) \cdot\left(H_{\mathrm{R}, n}\left(\psi_{\mathrm{R}, n}, \omega\right) \hat{\boldsymbol{p}}_{\mathrm{R}, n}\right)$

where $H_{\mathrm{R}, n}$ is the scalar reception pattern of the $n^{\text {th }}$ receiving antenna, $\psi_{\mathrm{R}, n}$ is the angle between the vector defining the antenna orientation and the vector from the antenna to the target, and $\hat{\boldsymbol{p}}_{\mathrm{R}, n}$ is the unit vector containing the horizontal and vertical components of the antenna polarization in the basis of the antenna. [11], [12].

The motion of the moving target, transmitters, and receivers included in expression (5) is modeled as in [8] by taking the inverse Fourier transform of the scattered electric field $\boldsymbol{E}_{m}^{\mathrm{r}}$ at position $\gamma_{\mathrm{R}, n}(s)$ to obtain an expression of the scattered electric field in terms of a fast-time variable $t$, substituting appropriate terms to account for the velocities of the movers in the scene, and taking the Fourier transform of the expression to again obtain a frequency-domain scattered electric field. This primarily results in a Doppler shift $\beta_{a} \omega_{m}$ where $\omega_{m}$ is the carrier frequency of the waveform transmitted by the $m^{\text {th }}$ transmitter,

$$
\begin{gathered}
\beta_{a}=\frac{\left(\boldsymbol{v}_{a}-\boldsymbol{v}_{\mathrm{T}, m}(s)\right) \cdot \hat{\boldsymbol{r}}_{\mathrm{T}, m}^{a}+\left(\boldsymbol{v}_{a}-\boldsymbol{v}_{\mathrm{R}, n}(s)\right) \cdot \hat{\boldsymbol{r}}_{\mathrm{R}, n}^{a}}{\mathrm{c}_{0}}, \\
\boldsymbol{r}_{\mathrm{R}, n}^{a}=\boldsymbol{x}_{a}-\gamma_{\mathrm{R}, n}(s),
\end{gathered}
$$

$\boldsymbol{v}_{a}$ is the velocity of the $a^{\text {th }}$ target, and $\boldsymbol{v}_{\mathrm{T}, m}(s)$ and $\boldsymbol{v}_{\mathrm{R}, n}(s)$ are the velocities of the $m^{\text {th }}$ transmitter and $n^{\text {th }}$ receiver at time $s$, respectively.
It is assumed that the signals received at the $n^{\text {th }}$ receiver and originating from the $m$ transmitters cannot be separated. The total time-domain data obtained at the $n^{\text {th }}$ receiver corresponding to the signal radiated from all transmitters and scattered by a distribution of point-like targets $p_{\mathrm{s}}\left(\boldsymbol{x}_{a}, \boldsymbol{v}_{a}\right)$ is written in terms of the time-domain received voltage as

$$
d_{n}(t)=\int \sum_{m} v_{m n}^{\mathrm{r}}\left(t, \boldsymbol{x}_{a}, \boldsymbol{v}_{a}\right) p_{\mathrm{s}}\left(\boldsymbol{x}_{a}, \boldsymbol{v}_{a}\right) \mathrm{d} \boldsymbol{x}_{a} \mathrm{~d} \boldsymbol{v}_{a}
$$

where the time-domain received voltage $v_{m n}^{\mathrm{r}}$ is the inverse Fourier transform of (5). Although the data model utilizes a target-centered coordinate system to define angles and relative positions of transmitters, receivers, and a target of interest, the expression for the total received data is represented in a global scene-centered coordinate system by defining positions relative to a chosen origin, requiring only a simple distanceand angle-preserving translation.

\section{CONCLUSION}

This work develops a data model for multistatic polarimetric radar with moving platforms and moving targets, consistent with an airborne MTI scenario.

\section{ACKNOWLEDGEMENT}

This work was sponsored by the Office of Naval Research under the Naval Research Laboratory Base Program.

\section{REFERENCES}

[1] R. S. Adve, R. A. Schneible, M. C. Wicks, and R. McMillan, "Spacetime adaptive processing for distributed aperture radars," in 2004 International Waveform Diversity and Design Conference, Edinburgh, UK, Nov. 2004, pp. 1-5.

[2] L. Landi and R. S. Adve, "Time-orthogonal-waveform-space-time adaptive processing for distributed aperture radars," in 2007 International Waveform Diversity and Design Conference, Pisa, IT, Jun. 2007, pp. 13-17.

[3] I. Bradaric, G. Capraro, D. Weiner, and M. Wicks, "Multistatic radar systems signal processing," in 2006 IEEE Radar Conference, Verona, New York, USA, Apr. 2006, p. 22.

[4] T. Varsolt, B. Yazici, and M. Cheney, "Wide-band pulse-echo imaging with distributed apertures in multi-path environments," Inverse Problems, vol. 24, no. 4, p. 045013, Aug. 2008.

[5] M. Cheney and B. Borden, "Imaging moving targets from scattered waves," Inverse Problems, vol. 24, p. 22, Oct. 2008.

[6] L. Wang, M. Cheney, and B. Borden, "Multistatic radar imaging of moving targets," IEEE Trans. on Aerospace and Electronic Systems, vol. 48, no. 1, pp. 230 - 242, Jan. 2012.

[7] T. Webster, L. Xu, and M. Cheney, "Antenna beam patterns in MIMO radar," in 2012 IEEE Radar Conference, Atlanta, GA, USA, May 2012, pp. 332-337.

[8] T. Webster, M. Cheney, and E. L. Mokole, "Multistatic polarimetric radar data modeling and imaging of moving targets," Inverse Problems, vol. 30, no. 3, p. 24, Feb. 2014.

[9] K. Voccola, M. Cheney, and B. Yazici, "Polarimetric synthetic-aperture inversion for extended targets in clutter," Inverse Problems, vol. 29, no. 5, p. 25, Apr. 2013.

[10] I. Son and B. Yazici, "Passive imaging with multistatic polarimetric radar," in 2012 IEEE Radar Conference, Arlington, VA, USA, May 2015, pp. 1584-1589.

[11] D. L. Sengupta and C.-T. Tai, "Radiation and reception of transients by linear antennas," in Transient Electromagnetic Fields, L. B. Felsen, Ed. Berlin: Springer-Verlag, 1976, ch. 4, pp. 181-235.

[12] S. J. Orfanidis, "Electromagnetic Waves and Antennas," www.ece. rutgers.edu/ orfanidi/ewa, Mar. 52012. 